\title{
Semi-analytical solution of Poisson's equation in bounded domain
}

\author{
H. Song ${ }^{1} \quad$ L. TaO $^{2}$ \\ (Received 2 March 2010; revised 21 April 2010)
}

\begin{abstract}
Poisson's equation is very important in electrostatics, mechanical engineering and theoretical physics. The novel semi-analytical, scaled boundary finite element method (SBFEM), is applied to solve Poisson's equation with Dirichlet and Neumann boundary conditions in the bounded domain. The SBFEM weakens the governing differential equation in the circumferential direction and solves the weakened equation analytically in the radial direction, combining the advantages of the finite element method and the boundary element method. Three examples demonstrate the excellent computational accuracy and efficiency of the SBFEM approach, revealing the great potential of this method to solve more complex engineering problems.
\end{abstract}

\section{Contents}

http://anziamj . austms.org.au/ojs/index.php/ANZIAMJ/article/view/2427 gives this article, (C) Austral. Mathematical Soc. 2010. Published May 3, 2010. ISSN 1446-8735. (Print two pages per sheet of paper.) 
2 Theoretical consideration

2.1 Governing equations . . . . . . . . . . . . . . C C172

2.2 Scaled boundary finite element transformation . . . . . . C C172

3 Solution procedure

4 Result and discussion

5 Conclusion

C183

References

C183

\section{Introduction}

Poisson's equation has many applications in electrostatics, mechanical engineering and theoretical physics. For example, the numerical solution of the incompressible Navier-Stokes equations involves solving the pressure Poisson's equation. Physical problems governed by the two dimensional Poisson's equation are often in a rectangular or circular domain, with Dirichlet, Neumann or mixed boundary conditions.

Poisson's equation is usually solved by some discretisation techniques such as the boundary element method (BEM) and the finite element method (FEM). BEM has the inherent advantage for problems in the unbounded domain with the property of reducing the spatial dimension by one. However, fundamental solutions are required and singular integrals exist. Furthermore, it suffers from the problems caused by irregular frequencies and sharp corners. On the other hand, FEM has a great advantage of a wide variety of element types. However, an associated boundary condition or an infinite element technique has to be introduced for problems in the unbounded domain. For three dimensional problems in the unbounded domain, the whole computation cost is much larger compared to BEM. 
Recently, a new semi-analytical method, namely the scaled boundary finite element method (SBFEM) has been successfully applied to soil-structure interaction, combining the advantages of both FEM and BEM [9]. The method only discretises the body boundary surface with finite elements, then transforms the governing partial differential equations to ordinary matrix differential equations in the radial direction which are then solved analytically. The method is more suitable for handling problems with singularities and is more efficient for solving problems of unbounded domains compared to FEM. Unlike BEM, it does not require a fundamental solution and is free from the irregular frequency difficulty. Fewer elements are needed to obtain a very accurate solution.

The SBFEM was first proposed to solve problems in soil-structure interaction by Song and Wolf [5], and later was applied to a variety of engineering fields. For example, Ekevid and Wiberg [2] analysed wave propagation related to moving loads in railway engineering by the combination of SBFEM and FEM. The dynamical response of a railroad section demonstrated the performance of the method. Teng et al. [8] simulated the water sloshing in a rectangular water container by SBFEM, finding that the SBFEM method gives much better results than the FEM method for the same mesh size. Li et al. [4] obtained a semi-analytical solution for the characteristics of a two dimensional dam-reservoir system with an absorptive reservoir bottom, in the frequency domain, by coupling SBFEM and FEM. Tao et al. [7] solved the problem of short-crested waves diffracted by a vertical circular cylinder using SBFEM, demonstrating high accuracy and efficiency in the unbounded domain. This was achieved with a small number of surface finite elements. Recently their solution was extended to water wave interaction with multiple cylinders of arbitrary shape [6].

A two dimensional Poisson's equation with Dirichlet and Neumann boundary conditions in a bounded domain is solved by SBFEM. Discretising only the boundary with surface finite elements, the current SBFEM model exhibits excellent computational accuracy and efficiency. SBFEM agrees well with analytical results and is obtained with low computational cost. 


\section{Theoretical consideration}

\subsection{Governing equations}

The two dimensional Poisson's equation in the following is the famous elliptic partial differential equation

$$
\nabla^{2} \phi(x, y)=-f(x, y), \quad \text { in } \Omega,
$$

where $\nabla$ is the Laplacian, $\phi(x, y)$ is the function to be solved, $f(x, y)$ is a known function, and $\Omega$ is the solution domain. Defining the Dirichlet boundary and Neumann boundary as $\Gamma_{\phi}$ and $\Gamma_{v}$ respectively, we have

$$
\begin{aligned}
& \phi=\bar{\phi}, \quad \text { on } \Gamma_{\phi}, \\
& \phi_{, \mathrm{n}}=\bar{\phi}_{\mathrm{n}}, \text { on } \Gamma_{v},
\end{aligned}
$$

where the overbar denotes a prescribed value, and a comma in the subscript designates the partial derivative with respect to the variable following the comma.

\subsection{Scaled boundary finite element transformation}

The finite element method requires the weighted residuals of the governing equation to be zero. Hence Equations (1), (2) and (3) are multiplied by a weighting function $w$ and integrated over the solution domain and the boundary. Performing integration by parts, the resulting equation becomes

$$
\int_{\Omega} \nabla^{\top} w \nabla \phi \mathrm{d} \Omega-\int_{\Omega} w f \mathrm{~d} \Omega-\oint_{\Gamma_{v}} w \bar{\phi}_{\mathrm{n}} \mathrm{d} \Gamma=0 .
$$

SBFEM defines the solution domain $\Omega$ by scaling a single piecewise smooth curve $S$ relative to a scaling centre $\left(x_{0}, y_{0}\right)$ (see Figure 1$)$. The circumferential 


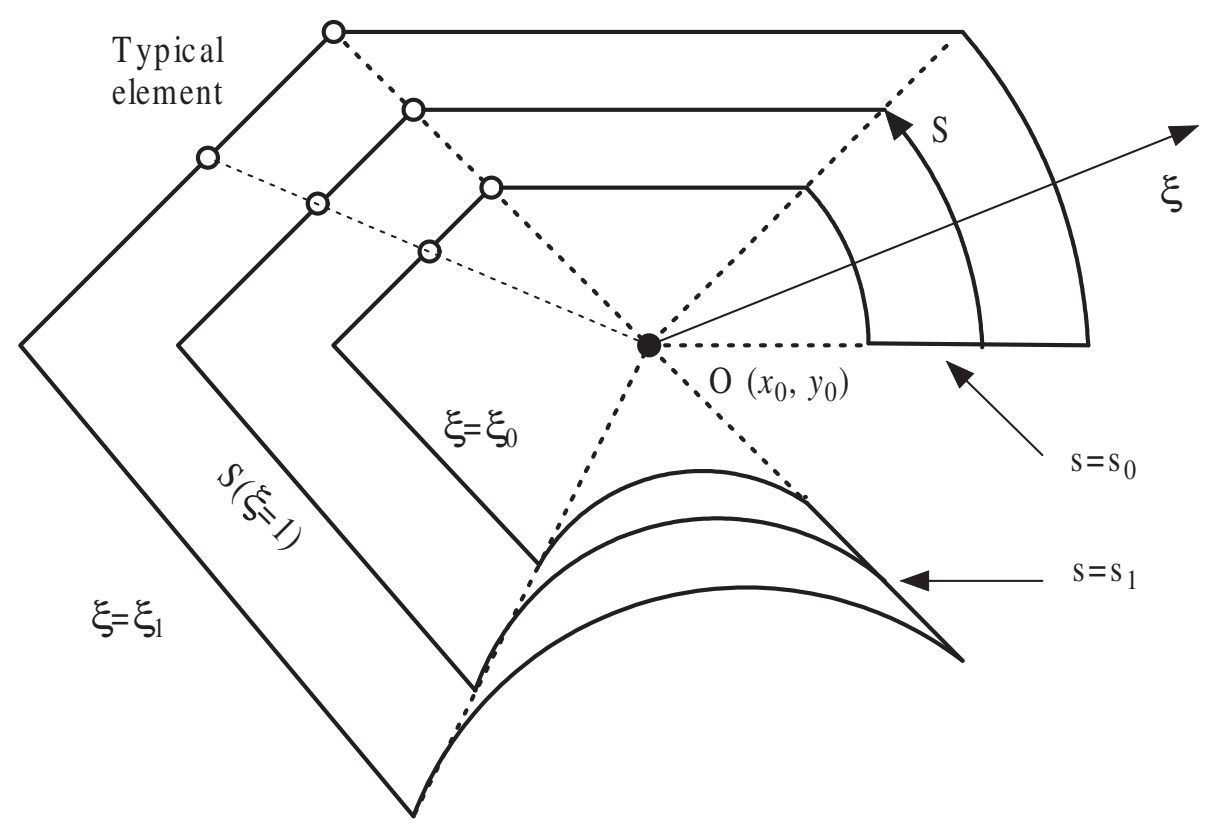

Figure 1: Definition of the coordinate system $(s, \xi)$ for SBFEM.

coordinate $\boldsymbol{S}$ is anticlockwise along the curve $S$ and the normalised radial coordinate $\xi$ is a scaling factor, defined as 1 at curve $S$ and 0 at the scaling centre. The whole solution domain $\Omega$ is in the range of $\xi_{0} \leqslant \xi \leqslant \xi_{1}$ and $s_{0} \leqslant s \leqslant s_{1}$. The two straight sections $s=s_{0}$ and $s=s_{1}$ are called side faces. They coincide when the curve $S$ is closed. For bounded domain, $\xi_{0}=0$ and $\xi_{1}=1$; whereas, for unbounded domain, $\xi_{0}=1$ and $\xi_{1}=\infty$. Therefore Cartesian coordinates are transformed to the scaled boundary coordinate $\xi$ and $s$ with the scaling equations

$$
x=x_{0}+\xi x_{s}(s), \quad y=y_{0}+\xi y_{s}(s) .
$$

By employing SBFEM, an approximate solution of $\phi$ is sought as

$$
\phi_{A}(\xi, s)=\boldsymbol{N}(s) \boldsymbol{a}(\xi),
$$


where $\boldsymbol{N}(\mathrm{s})$ is the shape function, the vector $\boldsymbol{a}(\xi)$ is analogous to the nodal values, the same as in FEM. The radial function $a_{j}(\xi)$ represents the variation of $\phi$ in the radial axis $\xi$ at each node $\boldsymbol{j}$, and the shape function $\boldsymbol{N}(\mathrm{s})$ interpolates between the nodal values in the circumferential axis $s$.

By performing the scaled boundary transformation [9], the operator

$$
\nabla=\boldsymbol{b}_{1}(\mathrm{~s}) \frac{\partial}{\partial \xi}+\frac{1}{\xi} \boldsymbol{b}_{2}(\mathrm{~s}) \frac{\partial}{\partial \mathrm{s}},
$$

where $\boldsymbol{b}_{1}(s)$ and $\boldsymbol{b}_{2}(s)$ depend only on the boundary

$$
\boldsymbol{b}_{1}(s)=\frac{1}{|J|}\left\{\begin{array}{c}
y_{s}(s)_{, s} \\
-x_{s}(s)_{, s}
\end{array}\right\}, \quad \boldsymbol{b}_{2}(s)=\frac{1}{|J|}\left\{\begin{array}{c}
-y_{s}(s) \\
x_{s}(s)
\end{array}\right\},
$$

and $|J|$ is the Jacobian at the boundary

$$
|J|=x_{s}(s) y_{s}(s)_{, s}-y_{s}(s) x_{s}(s)_{, s} .
$$

Applying the Galerkin approach [9], the weighting function $w$ is formulated using the same shape function as in Equation (6):

$$
\boldsymbol{w}(\xi, s)=\boldsymbol{N}(\mathrm{s}) \boldsymbol{w}(\xi)=\boldsymbol{w}(\xi)^{\top} \boldsymbol{N}(s)^{\mathrm{T}} .
$$

Substituting Equations (6), (7), and (10) into Equation (4) and integrating the terms containing $\boldsymbol{w}(\xi), \xi$ by parts with respect to $\xi$ using Green's theorem leads to

$$
\begin{gathered}
\boldsymbol{q}\left(\xi_{1}\right)=\int_{\mathrm{S}} \boldsymbol{N}(\mathrm{s})^{\mathrm{T}}\left(\bar{\nu}_{\mathrm{n}}\left(\xi_{1}, \mathrm{~s}\right)\right) \xi_{1} \mathrm{~d} s, \\
\boldsymbol{q}\left(\xi_{0}\right)=-\int_{\mathrm{S}} \boldsymbol{N}(\mathrm{s})^{\top}\left(\bar{v}_{\mathrm{n}}\left(\xi_{0}, \mathrm{~s}\right)\right) \xi_{0} \mathrm{~d} s,
\end{gathered}
$$

$$
\boldsymbol{E}_{0} \xi^{2} \boldsymbol{a}(\xi)_{, \xi \xi}+\left(\boldsymbol{E}_{0}+\boldsymbol{E}_{1}^{\mathrm{T}}-\boldsymbol{E}_{1}\right) \xi \boldsymbol{a}(\xi)_{, \xi}-\boldsymbol{E}_{2} \boldsymbol{a}(\xi)+\xi^{2} \boldsymbol{F}_{\mathrm{b}}=\xi \boldsymbol{F}_{\mathrm{s}}(\xi),
$$

where

$$
\mathrm{d} \Omega=|J| \xi \mathrm{d} \xi \mathrm{d} s, \quad \boldsymbol{q}(\xi)=\boldsymbol{E}_{0} \xi \boldsymbol{a}(\xi)_{, \xi}+\boldsymbol{E}_{1}^{\top} \boldsymbol{a}(\xi),
$$




$$
\begin{aligned}
& \boldsymbol{B}_{1}(\mathrm{~s})=\boldsymbol{b}_{1}(\mathrm{~s}) \boldsymbol{N}(\mathrm{s}), \quad \boldsymbol{B}_{2}(\mathrm{~s})=\boldsymbol{b}_{2}(\mathrm{~s}) \boldsymbol{N}(\mathrm{s})_{, \mathrm{s}}, \\
& \boldsymbol{E}_{0}=\int_{\mathrm{S}} \boldsymbol{B}_{1}(\mathrm{~s})^{\mathrm{T}} \boldsymbol{B}_{1}(\mathrm{~s})|J| \mathrm{ds}, \quad \boldsymbol{E}_{1}=\int_{\mathrm{S}} \boldsymbol{B}_{2}(\mathrm{~s})^{\mathrm{T}} \boldsymbol{B}_{1}(\mathrm{~s})|\mathrm{J}| \mathrm{d} \mathrm{s}, \\
& \boldsymbol{E}_{2}=\int_{\mathrm{S}} \boldsymbol{B}_{2}(\mathrm{~s})^{\mathrm{T}} \boldsymbol{B}_{2}(\mathrm{~s})|\mathrm{J}| \mathrm{d} \mathrm{s}, \quad \boldsymbol{F}_{\mathrm{b}}=\int_{\mathrm{S}} \boldsymbol{N}^{\mathrm{T}} \mathrm{f}|\mathrm{J}| \mathrm{d} \mathrm{s}, \\
& \boldsymbol{F}_{\mathrm{s}}(\xi)=\boldsymbol{N}\left(\mathrm{s}_{0}\right)^{\mathrm{T}}\left(-\bar{v}_{\mathrm{n}}\left(\xi, \mathrm{s}_{0}\right)\right)\left|\mathrm{J}\left(\mathrm{s}_{0}\right)\right| \\
& +\boldsymbol{N}\left(\mathrm{s}_{1}\right)^{\mathrm{T}}\left(-\bar{v}_{\mathrm{n}}\left(\xi, \mathrm{s}_{1}\right)\right)\left|\mathrm{J}\left(\mathrm{s}_{1}\right)\right| \text {. }
\end{aligned}
$$

Equation (13) is the scaled boundary finite element equation. By introducing the shape function, Poisson's equation has been weakened in the circumferential direction, so that the governing partial differential equation is transformed to an ordinary matrix differential equation in the radial direction. The rank of matrices $\boldsymbol{E}_{0}, \boldsymbol{E}_{1}, \boldsymbol{E}_{2}$ and vector $\boldsymbol{a}(\xi)$ is $\mathrm{m}$ (where $\mathrm{m}$ is the number of nodes in the curve $\boldsymbol{S}$ ). The nonzero term $\boldsymbol{F}_{\mathrm{b}}$ is caused by the nonhomogeneous term $f(x, y)$ in Equation (1), leading to the final governing equation Equation (13) becoming non-homogeneous. The presence of such a non-homogeneous term in the governing equation makes the solution procedure much more complicated than that of Tao et al. [7].

Boundary conditions are weakened in the form of Equations (11) and (12) respectively. For the boundary value problem in the unbounded region, $\xi_{0}=$ 1 on the boundary and $\xi_{1}=+\infty$ at infinity. For the boundary value problem in the bounded region, $\xi_{0}=0$ and $\xi_{1}=1$.

\section{Solution procedure}

The following procedure is rather different from earlier work of Tao et al. [7] due to the non-homogeneous term caused by the term $f(x, y)$ in Poisson's equation (1). However, the solution remains semi-analytical and fundamentalsolution-less in the following solution procedure, which is superior to many other numerical methods. 
Define

$$
\begin{aligned}
& \boldsymbol{X}(\xi)=\left\{\begin{array}{l}
\boldsymbol{a}(\xi) \\
\boldsymbol{q}(\xi)
\end{array}\right\}, \\
& \boldsymbol{F}(\xi)=\left\{\begin{array}{c}
0 \\
\xi^{2} \boldsymbol{F}_{\mathrm{b}}(\xi)-\xi \boldsymbol{F}_{\mathrm{s}}(\xi)
\end{array}\right\}, \\
& \boldsymbol{Z}=\left[\begin{array}{cc}
\boldsymbol{E}_{0}^{-1} \boldsymbol{E}_{1}^{\top} & -\boldsymbol{E}_{0}^{-1} \\
-\boldsymbol{E}_{2}+\boldsymbol{E}_{1} \boldsymbol{E}_{0}^{-1} \boldsymbol{E}_{1}^{\top} & -\boldsymbol{E}_{1} \boldsymbol{E}_{0}^{-1}
\end{array}\right] .
\end{aligned}
$$

Equation (13) is written as

$$
\zeta \boldsymbol{X}(\xi)_{, \xi}=-\boldsymbol{Z} \boldsymbol{X}(\xi)-\boldsymbol{F}(\xi) .
$$

The Hamiltonian matrix $\boldsymbol{Z}$ consists of two groups with opposite sign eigenvalues, $\boldsymbol{\Lambda}_{0}$ and $-\boldsymbol{\Lambda}_{0}$ [9]. The real parts of eigenvalues in $\boldsymbol{\Lambda}_{0}$ are all nonnegative. The eigenvalue problem is formulated as

$$
Z \boldsymbol{J}=-\boldsymbol{J} \boldsymbol{\Lambda} .
$$

Similar to previous research [5, 9], the analytical solution of Equation (22) is expressed as

$$
\begin{aligned}
& \boldsymbol{a}(\xi)=\boldsymbol{J}_{11} \xi^{-\boldsymbol{\Lambda}_{0}} \boldsymbol{C}_{1}(\xi)+\boldsymbol{J}_{12} \xi^{\boldsymbol{\Lambda}_{0}} \boldsymbol{C}_{2}(\xi), \\
& \boldsymbol{q}(\xi)=\boldsymbol{J}_{21} \xi^{-\boldsymbol{\Lambda}_{0}} \boldsymbol{C}_{1}(\xi)+\boldsymbol{J}_{22} \xi^{\boldsymbol{\Lambda}_{0}} \boldsymbol{C}_{2}(\xi),
\end{aligned}
$$

where

$$
\begin{aligned}
& \boldsymbol{C}_{1}(\xi)=\boldsymbol{c}_{1}-\int_{1}^{\xi} \zeta \cdot \zeta^{\boldsymbol{\Lambda}_{0}} \boldsymbol{A}_{12} \boldsymbol{F}_{\mathrm{b}} \mathrm{d} \xi, \\
& \boldsymbol{C}_{2}(\xi)=\boldsymbol{c}_{2}-\int_{1}^{\xi} \zeta \cdot \zeta^{-\boldsymbol{\Lambda}_{0}} \boldsymbol{A}_{22} \boldsymbol{F}_{\mathrm{b}} \mathrm{d} \xi, \\
& \boldsymbol{J}=\left[\begin{array}{ll}
\boldsymbol{J}_{11} & \boldsymbol{J}_{12} \\
\boldsymbol{J}_{21} & \boldsymbol{J}_{22}
\end{array}\right], \quad \boldsymbol{A}=\boldsymbol{J}^{-1}=\left[\begin{array}{ll}
\boldsymbol{A}_{11} & \boldsymbol{A}_{12} \\
\boldsymbol{A}_{21} & \boldsymbol{A}_{22}
\end{array}\right],
\end{aligned}
$$


and $\boldsymbol{c}_{1}$ and $\boldsymbol{c}_{2}$ are integration constants.

For the problem of a bounded domain, $\phi(\xi)$ at $\xi=0$ must remain finite, leading to $\left.\mathrm{C}_{2}(\xi)\right|_{\xi=0}=0$, then

$$
\begin{aligned}
& \boldsymbol{c}_{2}=\int_{1}^{0} \zeta \cdot \zeta^{-\boldsymbol{\Lambda}_{0}} \boldsymbol{A}_{22} \boldsymbol{F}_{\mathrm{b}} \mathrm{d} \xi, \\
& \begin{aligned}
\boldsymbol{C}_{2}(\xi) & =-\int_{0}^{\xi} \zeta \cdot \zeta^{-\boldsymbol{\Lambda}_{0}} \boldsymbol{A}_{22} \boldsymbol{F}_{\mathrm{b}} \mathrm{d} \xi, \\
\boldsymbol{a}(\xi) & =\boldsymbol{J}_{11} \xi^{-\boldsymbol{\Lambda}_{0}}\left(\boldsymbol{c}_{1}+\int_{\xi}^{1} \zeta^{-1} \zeta^{\boldsymbol{\Lambda}_{0}} \boldsymbol{A}_{12} \boldsymbol{F}_{\mathrm{b}} \mathrm{d} \xi\right) \\
& -\boldsymbol{J}_{12} \xi^{\boldsymbol{\Lambda}_{0}} \int_{0}^{\xi} \zeta^{-1} \zeta^{-\boldsymbol{\Lambda}_{0}} \boldsymbol{A}_{22} \boldsymbol{F}_{\mathrm{b}} \mathrm{d} \xi,
\end{aligned}
\end{aligned}
$$

$\boldsymbol{c}_{1}$ is determined by the boundary condition at $\xi=1$

$$
\begin{gathered}
\left.\boldsymbol{a}(\xi)\right|_{\xi=1}=\boldsymbol{J}_{11} \boldsymbol{c}_{1}-\boldsymbol{J}_{12} \int_{0}^{1} \zeta^{-1} \zeta^{-\boldsymbol{\Lambda}_{0}} \boldsymbol{A}_{22} \boldsymbol{F}_{\mathrm{b}} \mathrm{d} \xi \\
\left.\boldsymbol{q}(\xi)\right|_{\xi=1}=\boldsymbol{J}_{21} \boldsymbol{c}_{1}-\boldsymbol{J}_{22} \int_{0}^{1} \zeta^{-1} \zeta^{-\boldsymbol{\Lambda}_{0}} \boldsymbol{A}_{22} \boldsymbol{F}_{\mathrm{b}} \mathrm{d} \xi
\end{gathered}
$$

For the problem of the unbounded domain, the solution procedure is similar.

\section{Result and discussion}

Three examples were calculated to demonstrate the excellent computational accuracy and efficiency of the proposed SBFEM approach. The first example is the well known 'Kirchoff vortices' [3]. A Kirchoff vortex is an ellipse of constant vorticity $\omega$, with major axis $2 a$, minor axis $2 b$. The rotation of the major axis of the ellipse is due to the phase speed of the perturbation along the vortex contour and to the advection by the mean azimuthal velocity of 


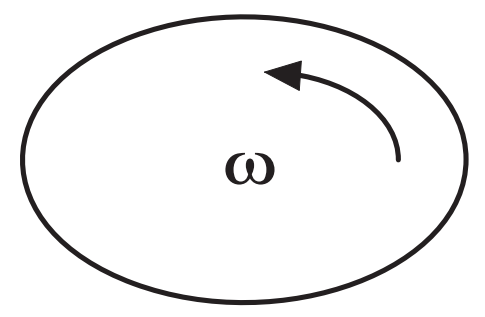

FiguRE 2: Kirchoff vortex

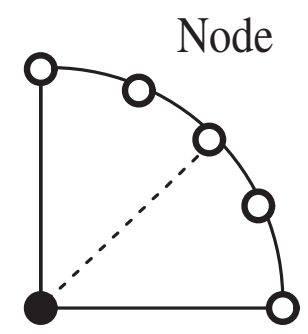

Scaling centre

Figure 3: Scaled boundary finite element mesh for Example 1.

the vortex (see Figure 2). A Kirchoff ellipse is stable for $b / a>1 / 3$ [1]. For simplicity, we choose $b=a$ in this paper, and the vortex is centered at the origin $\left(x_{0}, y_{0}\right)$. So, $\phi(x, y)$ in Equation (1) is the stream function and $f(x, y)$ in Equation (1) is the vorticity $\omega$ in this case. The scaling centre is also chosen at the origin, and the body boundary is discretised with three node quadratic elements along the circumference. Due to the symmetry of the physical problem, only a quarter of the boundary needs to be discretised.

Table 1 shows the values of nondimensional normal derivative of the streamfunction $\phi_{, n} /(\omega \mathrm{a})$ on the boundary, calculated using the SBFEM, where the Dirichlet boundary condition was given as $\bar{\phi}=-\omega a^{2} / 4$ on the edge of the ellipse. As shown in the table, even one element obtains the same result as 
TABLE 1: Nondimensional normal derivative of the streamfunction on the edge of the ellipse.

\begin{tabular}{ll}
\hline & $\phi_{, \mathrm{n}} /(\omega \mathrm{a})$ \\
\hline 1 element & -0.5000000 \\
2 element & -0.5000000 \\
3 element & -0.5000000 \\
Exact & -0.5 \\
\hline
\end{tabular}

TABLE 2: Nondimensional streamfunction $\phi /\left(\omega \mathrm{a}^{2}\right)$ at different locations.

\begin{tabular}{llllll}
\hline $\mathrm{r} / \mathrm{a}$ & $1 / 4$ & $1 / 3$ & $1 / 2$ & $2 / 3$ & $3 / 4$ \\
\hline 1 element & -0.015625 & -0.027778 & -0.062500 & -0.111111 & -0.140625 \\
2 element & -0.015625 & -0.027778 & -0.062500 & -0.111111 & -0.140625 \\
3 element & -0.015625 & -0.027778 & -0.062500 & -0.111111 & -0.140625 \\
Exact & -0.015625 & -0.027778 & -0.0625 & -0.111111 & -0.140625 \\
\hline
\end{tabular}

the exact analytical solution. Table 2 lists the nondimensional stream function $\phi /\left(\omega \mathrm{a}^{2}\right)$, calculated at different locations within the bounded domain $r / a=1 / 4,1 / 3,1 / 2,2 / 3$ and 3/4. Excellent agreement is achieved with only one element. This demonstrates the superiority of SBFEM.

The second example is in a bounded square region $0 \leqslant x, y \leqslant 1$. The nonhomogeneous term $f(x, y)=2 x^{3}-6 x y(1-y)$ and the boundary conditions are the Dirichlet boundary conditions

$$
\left\{\begin{array}{l}
\phi(0, y)=0 \\
\phi(1, y)=y(1-y) \\
\phi(x, 0)=0 \\
\phi(x, 1)=0
\end{array}\right.
$$

The problem has the exact solution $\phi(x, y)=x^{3} y(1-y)$.

Figure 5 compares the SBFEM result and the analytical solution for Example 2 . With only 16 three node quadratic elements along one side (33 nodes in 


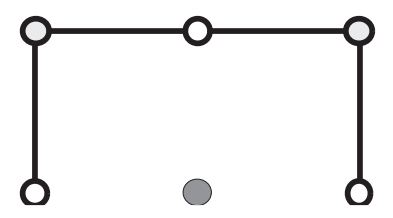

\section{- Node \\ Scaling centre}

FiguRE 4: Scaled boundary finite element mesh for Examples 2 and 3.

one side), the method achieved very good agreement with the analytical solution, while more elements are required for traditional discretisation method such as finite difference methods. Due to the symmetry of the problem, only half of the circumference needs to be discretised (see Figure 4).

The third example is also in a square region $0 \leqslant x, y \leqslant 1$. The nonhomogeneous term $f(x, y)=2\left(2 y^{3}-3 y^{2}+1\right)+6\left(1-x^{2}\right)(1-2 y)$ and the boundary conditions are the mixed boundary conditions

$$
\left\{\begin{array}{l}
\phi(0, y)=2 y^{3}-3 y^{2}+1 \\
\phi(1, y)=0 \\
\phi_{y}(x, 0)=0 \\
\phi_{y}(x, 1)=0
\end{array}\right.
$$

The exact solution of the problem is $\phi(x, y)=\left(1-x^{2}\right)\left(2 y^{3}-3 y^{2}+1\right)$.

Figure 6 compares the SBFEM result and the analytical solution for Example 3. Again, the figure shows that SBFEM results agree with the analytical solution at a very low computational cost. The discretisation is shown in Figure 4 as well. 



Figure 5: Comparison of the SBFEM result and the analytical solution for Example 2. 



Figure 6: Comparison of the SBFEM result and the analytical solution for Example 3. 
As solving the pressure Poisson's equation is the most time consuming task in viscous fluid solvers, the application of SBFEM in solving Poisson's equation will improve significantly computational efficiency and accuracy due to its semi-analytic nature. The methodology and theoretical approach presented in this article is an important step and will significantly reduce the computational cost of solving problems in viscous flow.

\section{Conclusion}

The semi-analytical scaled boundary finite element method is further extended and successfully applied to solve Poisson's equation with Dirichlet and Neumann boundary conditions in bounded domain. Excellent agreement between the present SBFEM results and the analytical solutions are achieved at a very low computational cost, demonstrating significant computational accuracy and efficiency - a distinct advantage over the existing methods for engineering problems. As many of the problems in ocean engineering are governed by Laplace's equation and Poisson's equation, the method presented in this article could lead to direct engineering applications.

Acknowledgement The first author is grateful for the postdoctoral fellowship at Griffith University to support this research.

\section{References}

[1] Carton, X., Hydrodynamical modeling of oceanic vortices, Surv. Geoph., 22, 2001, 179-263. doi:10.1023/A:1013779219578. C178

[2] Ekevid, T. and Wiberg, N-E., Wave propagation related to high-speed train: A scaled boundary FE-approach for unbounded domains, 
Comput. Methods Appl. Mech. Engrg., 191, 2002, 3947-2964. doi:10.1016/S0045-7825(02)00341-9. C171

[3] Kirchhoff, G., Vorlesungen über mathematische Physik, In Mechanik, B.G. Teubner, Leipzig, 1876.

http://www. archive.org/stream/vorlesungenberm02kircgoog. C177

[4] Li, S., Liang, H., and Li, A., A semi-analytical solution for characteristics of a dam-reservoir system with absorptive reservoir bottom, Journal of Hydrodynamics, Ser. B, 20, 2008, 727-734. doi:10.1016/S1001-6058(09)60008-1. C171

[5] Song, C., Wolf, J. P., The scaled boundary finite-element method-alias consistent infinitesimal finite-element cell method-for elastodynamics, Comput. Methods Appl. Mech. Engrg., 147, 1997, 329-355. doi:10.1016/S0045-7825(97)00021-2. C171, C176

[6] Song, H., Tao, L., and Chakrabarti, S., Modelling of water wave interaction with multiple cylinders of arbitrary shape, J. Comput. Phys., 229, 2010, 1498-1513. doi:10.1016/j.jcp.2009.10.041. C171

[7] Tao, L., Song, H. and Chakrabarti, S., Scaled boundary FEM solution of short-crested wave diffraction by a vertical cylinder, Comput. Method Appl. M., 197, 2007, 232-242. doi:10.1016/j.cma.2007.07.025. $\mathrm{C} 171, \mathrm{C} 175$

[8] Teng, B., Zhao, M., and He, G. H., Scaled boundary finite element analysis of the water sloshing in 2D containers, Int. J. Numer. Meth. Fluids, 52, 2006, 659-678. doi:10.1002/fld.1194. C171

[9] Wolf, J. P., The scaled boundary finite element method, John Wiley \& Sons Ltd, Chichester, England, 2003. http://au.wiley.com/ WileyCDA/WileyTitle/productCd-0471486825.html. C171, C174, $\mathrm{C} 176$ 


\section{Author addresses}

1. H. Song, Griffith School of Engineering, Griffith University, Queensland 4222, Australia.

mailto:h.song@griffith.edu.au

2. L. Tao, School of Marine Science and Technology, Newcastle University, NE1 7RU, England, UK. 